Gynäkologische Endokrinologie 2013 · 11:5-5 DOI 10.1007/s10304-012-0494-6

Online publiziert: 5. Januar 2013

(c) Springer-Verlag Berlin Heidelberg 2013

\section{T. Strowitzki}

Gynäkologische Endokrinologie und Fertilitätsstörungen, Universitätsfrauenklinik Heidelberg, Heidelberg
Liebe Leserinnen und Leser,

Transition von der pädiatrischen zur gynäkologischen Endokrinologie ist das neue Schlagwort. Darunter verstehen wir den kontinuierlichen Übergang der Betreuung endokrinologischer Patientinnen vom Kinderarzt zum Frauenarzt.

Die pädiatrische Endokrinologie hat herausragende Aufgaben in der Diagnostik und Therapie der Kinder mit endokrinen Störungen, der Pathologie der Pubertät und des Wachstums, sei es akzeleriert oder verlangsamt. Viele der schon in der Kindheit auffallenden endokrinen Störungen bedürfen einer lebenslangen Betreuung. Aus Mädchen werden junge Frauen. Fragen zur Zyklusentwicklung, zur Fruchtbarkeit und zur Kontrazeption stellen uns vor neue Herausforderungen.

\ Eine kontinuierliche Zusammenarbeit pädiatrischer und gynäkologischer Endokrinologen ist unerlässlich.

Gerade daran mangelt es aber vielerorts, und so gehen selbst junge erwachsene Frauen weiter zum Kinderendokrinologen ihres Vertrauens.

Die Universität Heidelberg hat eine Transitionssprechstunde eingerichtet. So wird die kontinuierliche Weiterbetreuung der erwachsen werdenden Patientinnen sichergestellt. Um dies gewährleisten zu können, muss der gynäkologische Endokrinologe aber über Grundkenntnisse der wesentlichen Probleme verfügen, die sich bei Kindern in endokrinologischer Betreuung stellen. Umgekehrt muss auch der pädiatrische Endokrinologe eine Vor- stellung von den Aufgaben der gynäkologischen Endokrinologie in der Betreuung der Jugendlichen entwickeln, z. B. hinsichtlich der Kontrazeption.

Genau hier greift die vorliegende Ausgabe von Gynäkologische Endokrinologie an. Wir konnten herausragende Referenten sowohl aus der Pädiatrie als auch aus der Gynäkologie gewinnen, die die führenden Meinungsbildner zu diesen Themen in Deutschland sind. Die Herausgeber erfreut dies umso mehr, als es zeigt, dass die Gynäkologische Endokrinologie mit ihrer profunden Arbeit an aktuellen Leitthemen ein hohes Ansehen genießt. Auch die juristischen Aspekte sind für die tägliche Praxis wesentlich.

Zunächst stellt $H$. Dörr aus Erlangen die Grundlagen der endokrinen Regulation der Pubertät dar. Im Fokus steht hier die zentrale Aktivierung des Gonadotropin-Releasing-Hormon(GnRH)-Pulsgenerators. Im zweiten Beitrag zeigen dann I. Baus u. P.-M. Holterhus aus Kiel, wie schwierig es oft ist, die vorzeitige $\mathrm{Pu}$ bertätsentwicklung von pathologischen Verläufen der pubertären Entwicklung zu unterscheiden. Gleiches gilt für die gar nicht so seltene konstitutionelle Entwicklungsverzögerung. D. Choukair und M. Bettendorf aus Heidelberg erläutern die Zusammenhänge zwischen Hormonen und Größenwachstum, zeigen aber auch, dass nicht alles hormonbedingt ist und das Größenwachstum in besonderem Maße auch vom Ernährungszustand und der Gesundheit des Kindes abhängt.

Das wichtige Thema der Kontrazeption bei Jugendlichen wird von M. Goeckenjan u. T. Rabe aus Heidelberg gemeinsam mit
E. Merkle aus Bad Reichenhall erläutert. Die Beratung und Behandlung von Jugendlichen steht an der Schnittstelle zwischen Eigenverantwortung der Jugendlichen und elterlichen Entscheidungen. Hier sind die Klarstellungen von Rechtsanwältin C. Halstrick aus München von enormer praktischer Relevanz.

Die Adipositas nimmt langsam ein endemisches Ausmaß an. B. Lennerz u. $M$. Wabitsch aus Ulm zeigen uns, welche gravierenden Folgen sie schon in der $\mathrm{Pu}$ bertät hat. Adipositas kann u. a. zu Störungen der Pubertätsentwicklung und begleitenden endokrinen und metabolischen Langzeitveränderungen führen.

Ich hoffe, dass die Themenwahl sowohl für Gynäkologen als auch für Pädiater von großem Wert ist und das Bewusstsein für die garantierte Betreuung unserer jungen Patientinnen schärft.

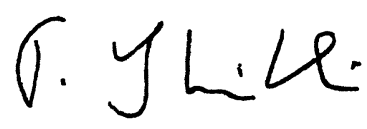

T. Strowitzki

\section{Korrespondenzadresse}

Prof. Dr. T. Strowitzki

Gynäkologische Endokrinologie und Fertilitätsstörungen,

Universitätsfrauenklinik Heidelberg

Voßstr. 9, 69115 Heidelberg

thomas.strowitzki@med.uni-heidelberg.de 